\title{
Simple J-factors and D-factors for indirect dark matter detection
}

\author{
N. W. Evans, ${ }^{1, *}$ J. L. Sanders, ${ }^{1, \dagger}$ and Alex Geringer-Sameth ${ }^{2, ;}$ \\ ${ }^{1}$ Institute of Astronomy, Madingley Rd, Cambridge CB3 OHA, United Kingdom \\ ${ }^{2}$ McWilliams Center for Cosmology, Department of Physics, Carnegie Mellon University, \\ Pittsburgh, Pennsylvania 15213, USA
}

(Received 28 January 2016; published 12 May 2016)

\begin{abstract}
J-factors (or D-factors) describe the distribution of dark matter in an astrophysical system and determine the strength of the signal provided by annihilating (or decaying) dark matter respectively. We provide simple analytic formulas to calculate the J-factors for spherical cusps obeying the empirical relationship between enclosed mass, velocity dispersion and half-light radius. We extend the calculation to the spherical Navarro-Frenk-White model, and demonstrate that our new formulas give accurate results in comparison to more elaborate Jeans models driven by Markov chain Monte Carlo methods. Of the known ultrafaint dwarf spheroidals, we show that Ursa Major II, Reticulum II, Tucana II and Horologium I have the largest J-factors and so provide the most promising candidates for indirect dark matter detection experiments. Amongst the classical dwarfs, Draco, Sculptor and Ursa Minor have the highest J-factors. We show that the behavior of the J-factor as a function of integration angle can be inferred for general dark halo models with inner slope $\gamma$ and outer slope $\beta$. The central and asymptotic behavior of the J-factor curves are derived as a function of the dark halo properties. Finally, we show that models obeying the empirical relation on enclosed mass and velocity dispersion have J-factors that are most robust at the integration angle equal to the projected half-light radius of the dwarf spheroidal (dSph) divided by heliocentric distance. For most of our results, we give the extension to the $\mathrm{D}$-factor which is appropriate for the decaying dark matter picture.
\end{abstract}

DOI: 10.1103/PhysRevD.93.103512

\section{INTRODUCTION}

The dwarf galaxies surrounding the Milky Way are the most extreme dark matter dominated objects known to us with central mass to light ratios typically of the order of tens to hundreds (e.g., [1,2]). Additionally, little or no emission has been detected in wavebands other than the optical, and so the intrinsic astrophysical backgrounds are low. This makes the dwarf spheroidals (dSphs) attractive targets to look for signals of dark matter annihilation products [3-6].

The $\gamma$-ray differential flux from dark matter annihilation measured within a solid angle $\Delta \Omega$ is (see e.g., [5,7])

$$
\frac{d \phi_{\gamma}}{d E_{\gamma}}=\phi^{\mathrm{PP}}(E) \times \mathrm{J}(\Delta \Omega) .
$$

The first term on the right-hand side depends on the particle physics (i.e. dark matter particle mass, annihilation cross section, and Standard Model final states). The second term is the astrophysical factor, or J-factor, and encapsulates the distribution of dark matter within the system of interest:

$$
\mathrm{J}=\iint \rho_{\mathrm{DM}}{ }^{2}(\ell, \Omega) d \ell d \Omega
$$

It is therefore the square of the dark matter density integrated along the line of sight and over the solid angle of the sky corresponding to the observation.

\footnotetext{
"nwe@ast.cam.ac.uk

jls@ast.cam.ac.uk

*alexgs@cmu.edu
}

For the dSphs, the dark matter density is not known, but can be constrained from the line-of-sight velocities of individual stars. The spherical Jeans equations are often used to relate the velocities of the stars to the underlying dark matter distribution. Nowadays, this is often explored in a Bayesian framework using Monte Carlo techniques, so that the calculation of the J-factors requires significant computational effort [8-13].

However, it is reasonable to look for a simpler way of computing J-factors for two reasons. First, for many of the recently discovered ultrafaint dwarf galaxies, there are few stars with line-of-sight velocities. In some cases, such as Ursa Major II, the giant branch itself is so sparse that there are very few target stars for spectroscopy [14]. Given such fundamental limitations on the observational data, the extensive exploration of model space in conventional Jeans analyses may be needlessly elaborate. Second, an entirely characteristic feature of the $\mathrm{dSphs}$ is that they are flattened. In fact, some of the ultrafaints are very highly flattened with ellipticities exceeding 0.5 , such as Ursa Major II [14], Hercules [15], Ursa Major I [16] and Reticulum II [17]. Therefore, the underlying physical model of a spherical dark halo containing a round distribution of stars satisfying the Jeans equation may fail to capture important aspects of the physics. This leaves the value of computationally intensive approaches based on sphericity open to question, as they may suffer from systematic effects when applied to flattened or triaxial systems. While there have already been some investigations 
of axisymmetry and triaxiality $[11,18]$, the implications of these effects on the dark matter annihilation signal warrants a systematic study, which we provide elsewhere [19].

The question as to whether dSphs have cored or cusped dark matter densities has been investigated intensively over the past few years. The latest simulations have reached a resolution where the effect of individual supernovae can be modeled, making them much less sensitive to the details of the "subgrid" star formation and feedback physics [20,21]. They suggest that dark matter cores of size comparable to the half-light radius of the stellar component should be present in objects like Carina and Fornax that have formed stars for a Hubble time. However, dSphs with patchy star formation may be more cuspy, with the ultrafaints possibly retaining a pristine cusp of the form originally proposed by Navarro, Frenk and White (NFW) [22]. This picture is broadly consistent with simple energy constraints [23], as well as tidal arguments suggesting that if the ultrafaints had dark matter cores, then they would not survive for long on their current orbits [24]. All this suggests that, even if the larger classical dSphs are cored as implied by the current observational data on general grounds [25], the smaller dSphs and the ultrafaints are probably cusped with dark matter densities behaving like $\rho_{\mathrm{DM}} \sim r^{-1}$ at small radii.

Here, we provide a set of simple formulas for J-factors in spherical cusped geometries. Our approach is entirely elementary, but we show that it leads to formulas that are very competitive with more laborious approaches. Section II concentrates on the cosmologically motivated $1 / r$ cusp exemplified by the NFW model. We provide an analytic formula for the J-factor of the NFW model, as well as new estimates for the classical and ultrafaint dwarfs, including the newly discovered Horologium I, Grus I, Hydra II, and Pisces II. Section III extends the work into more general cusped and cored dark haloes and explores the possibility of inferring halo structure from $\mathrm{J}$-factor profiles. In particular, we show how the behavior of the J-factor at small and large integration angles is controlled by the halo parameters. Finally, we investigate the existence of a sweet spot-namely an integration angle at which the value of the J-factor is reasonably insensitive to the unknowable aspects of the underlying dark halo profile $[9,10]$.

\section{SIMPLE FORMULAS FOR J-FACTORS}

Here, we provide a simple calculation for the J-factor of both a spherical cusp and a NFW halo. We show our formulas reproduce the results of more sophisticated calculations.

\section{A. Spherical cusp}

In spherical symmetry, dSphs follow the empirical law

$$
M_{\mathrm{h}}=M\left(R_{\mathrm{h}}\right) \approx \frac{2.5}{G} \sigma_{\operatorname{los}}^{2} R_{\mathrm{h}},
$$

where $R_{\mathrm{h}}$ is the (projected) half-light radius of the stars and $\sigma_{\text {los }}$ is the luminosity weighted square of the velocity dispersion. Specifically, this is defined as

$$
\sigma_{\mathrm{los}}^{2}=\frac{1}{L} \int_{V} \nu(r) v_{\mathrm{los}}^{2} d V
$$

where $L$ is the total luminosity, $\nu(r)$ is the luminosity density of the dSph and $v_{\text {los }}$ is the line-of-sight velocity. In other words, the physical content of Eq. (3) is that the mass enclosed within the half-light radius is robust against changes in anisotropy. This result was demonstrated from solutions of the spherical Jeans equations [26,27]. There are known biases in this formula when applied to populations deeply embedded with dark haloes, such as the metal-rich subpopulations of dSphs [28]. In our application to the entirety of the stellar population of the $\mathrm{dSph}$, any such bias is negligible, especially compared to the uncertainty in the velocity dispersion itself, which dominates the overall error budget.

Infinite spherical cusps obeying this law have enclosed mass

$$
M(r)=M_{\mathrm{h}}\left(\frac{r}{R_{\mathrm{h}}}\right)^{3-\gamma}=\frac{2.5 \sigma_{\text {los }}^{2}}{G R_{\mathrm{h}}^{2-\gamma}} r^{3-\gamma},
$$

where $0<\gamma<3$. The dark matter density is

$$
\rho_{\mathrm{DM}}(r)=\frac{M_{\mathrm{h}}}{4 \pi R_{\mathrm{h}}^{3-\gamma}} \frac{3-\gamma}{r^{\gamma}}=\frac{5 \sigma_{\mathrm{los}}^{2}}{8 \pi G R_{\mathrm{h}}^{2-\gamma}} \frac{3-\gamma}{r^{\gamma}} .
$$

This gives us a one-parameter family of dark matter cusps that always obey the empirical law (3).

We now make two assumptions to enable us to perform the integration in the J-factor analytically. First, we assume that the dSph is sufficiently distant that

$$
d \Omega d \ell \rightarrow \frac{1}{D^{2}} 2 \pi R d R d z
$$

Here, $D$ is the heliocentric distance, $z$ is the line of sight and $R$ is a polar coordinate in the plane of the sky. This approximation means that the projection is from infinity rather than from a finite distance. The integration volume is a cylinder rather than a cone. As even the nearest ultrafaints (Segue 1 and Reticulum II) are $\sim 20-30 \mathrm{kpc}$ distant, this incurs little actual error. Second, we assume that the $\mathrm{J}$-factor is dominated by contributions from the singular cusp. We show a posteriori that this approximation is fine by comparing our formula to the results of more elaborate calculations. 
The integration in Eq. (2) can now be done analytically

$$
\begin{aligned}
\mathrm{J} & =\frac{2 \pi}{D^{2}} \int_{-\infty}^{\infty} d z \int_{0}^{D \theta} R \rho_{\mathrm{DM}^{2}} d R \\
& =\frac{25 \sigma_{\mathrm{los}}^{4}}{64 G^{2}} \frac{1}{D^{2} R_{\mathrm{h}}}\left(\frac{D \theta}{R_{\mathrm{h}}}\right)^{3-2 \gamma} P(\gamma),
\end{aligned}
$$

where $P(\gamma)$ is a dimensionless number depending on the cusp slope $\gamma$

$$
P(\gamma)=\frac{2}{\pi^{1 / 2}} \frac{(3-\gamma)^{2} \Gamma\left(\gamma-\frac{1}{2}\right)}{(3-2 \gamma) \Gamma(\gamma)} .
$$

Here, $\Gamma(x)$ is the gamma function, whilst we require $1 / 2<\gamma<3 / 2$ for convergence. This constraint can be interpreted physically. If the density is too shallow $(\gamma<1 / 2)$, then the dark matter extends too far along the line of sight, causing the $z$ integral to diverge. Similarly, if it is too strongly cusped $(\gamma>3 / 2)$, then the contribution from the cusp at $r=0$ also causes the $z$ integral to diverge.

The angular integration is usually performed out to $\theta=0.5^{\circ}$ from the center of the $\mathrm{dSph}$, as this is typical of the resolution of Fermi-LAT data in the GeV range. Walker et al. [10] have argued that the J-factor is most robust to modeling uncertainty when the integration angle is $\theta_{\text {crit }} \approx 2 R_{\mathrm{h}} / D$, or twice the half-light radius of the stars divided by distance to the $\mathrm{dSph}$. Finally, it is also useful to compute the J-factor out to $\theta_{\max }=\operatorname{asin}\left(r_{\max } / D\right)$ where $r_{\max }$ is an estimate of the maximum galactocentric distance in the sample of observed member stars (see [12], Sec. 6. 2). We expect our formula to break down at large $\theta$, but this is usually comparable or beyond $\theta_{\max }$.

In scenarios in which the dark matter decays (rather than annihilates) to give $\gamma$ rays [29], it is also helpful to have the D-factor, which is just

$$
\mathrm{D}=\iint \rho_{\mathrm{DM}}(\ell, \Omega) d \ell d \Omega .
$$

Using the same approximation of an infinite cusp obeying the empirical law (3), we find

$$
\mathrm{D}=\frac{5 \sigma_{\text {los }}^{2}}{8 G} \frac{R_{\mathrm{h}}}{D^{2}}\left(\frac{D \theta}{R_{\mathrm{h}}}\right)^{3-\gamma} Q(\gamma),
$$

where

$$
Q(\gamma)=2 \pi^{1 / 2} \frac{\Gamma\left(\frac{\gamma}{2}-\frac{1}{2}\right)}{\Gamma(\gamma / 2)}
$$

with $1<\gamma<3$ for convergence. This of course does not converge for $\gamma=1$ because of contributions at large radii where the integral diverges logarithmically.

\section{B. NFW cusp}

There is ample numerical evidence that dark matter halos have an approximate double power-law structure ([22], hereafter NFW) with cusp slope $\gamma=1$. For this astrophysically important case, we obtain

$$
\mathrm{J}=\frac{25}{8 G^{2}} \frac{\sigma_{\mathrm{los}}^{4} \theta}{D R_{\mathrm{h}}^{2}} .
$$

This very simple result does not appear to have been given before. The D-factor however is infinite, as the surface density of an untruncated $1 / r$ density cusp does not converge. In fact, for the full NFW model, it is possible to carry out the integration explicitly for both the J- and the D-factors and obtain exact results. These are a little more cumbersome than the pure power-law case, but still simple enough to be useful.

We begin by introducing an auxiliary function (e.g., [30,31])

$$
X(s)= \begin{cases}\frac{1}{\sqrt{1-s^{2}}} \operatorname{Arcsech} s, & 0 \leq s \leq 1, \\ \frac{1}{\sqrt{s^{2}-1}} \operatorname{Arcsec} s, & s \geq .\end{cases}
$$

We note that $X(1)=1$ so that the function is continuous. We take the NFW model in the form

$$
\rho_{\mathrm{DM}}(r)=\frac{\rho_{0} r_{\mathrm{s}}^{3}}{r\left(r+r_{\mathrm{s}}\right)^{2}}
$$

Then, the J-factor is

$$
\begin{aligned}
\mathrm{J}= & \frac{\pi \rho_{0}^{2} r_{\mathrm{s}}^{2}}{3 D^{2} \Delta^{4}}\left[2 D \theta\left(7 D r_{\mathrm{s}}^{3} \theta-4 D^{3} r_{\mathrm{s}} \theta^{3}+3 \pi \Delta^{4}\right)\right. \\
& \left.+\frac{6}{r_{\mathrm{s}}}\left(2 \Delta^{6}-2 r_{\mathrm{s}}^{4} \Delta^{2}-D^{4} r_{\mathrm{s}}^{2} \theta^{4}\right) X\left(\frac{D \theta}{r_{\mathrm{s}}}\right)\right],
\end{aligned}
$$

Then, defining $y=D \theta / r_{\mathrm{s}}$, the $\mathrm{J}$-factor is

$$
\begin{aligned}
\mathrm{J}= & \frac{\pi \rho_{0}^{2} r_{\mathrm{s}}^{3}}{3 D^{2} \Delta^{4}}\left[2 y\left(7 y-4 y^{3}+3 \pi \Delta^{4}\right)\right. \\
& \left.+6\left(2 \Delta^{6}-2 \Delta^{2}-y^{4}\right) X(y)\right]
\end{aligned}
$$

where $\Delta^{2}=1-y^{2}=1-D^{2} \theta^{2} / r_{\mathrm{s}}^{2}$. Given a mass $M_{\mathrm{h}}$ enclosed within the half-light radius $R_{\mathrm{h}}$ the parameter $\rho_{0}$ is given by

$$
\rho_{0}=\frac{M_{\mathrm{h}}}{4 \pi r_{\mathrm{s}}^{3}}\left(\log \left[\frac{r_{\mathrm{s}}+R_{\mathrm{h}}}{r_{\mathrm{s}}}\right]-\frac{R_{\mathrm{h}}}{r_{\mathrm{s}}+R_{\mathrm{h}}}\right)^{-1} .
$$

On identifying $\rho_{0} r_{\mathrm{s}}=5 \sigma_{\mathrm{los}}^{2} /\left(4 \pi R_{\mathrm{h}} G\right)$ and letting $r_{\mathrm{s}} \rightarrow \infty$, we obtain the infinite cusp (6) with $\gamma=1$. In this limit, the full J-factor (16) reduces to (13), as it should. In the other 
limit, as $\theta$ becomes large, the $\mathrm{J}$-factor curve turns over and tends to the asymptotic value

$$
\mathrm{J} \rightarrow \frac{4 \pi}{3} \frac{\rho_{0}^{2} r_{\mathrm{s}}^{3}}{D^{2}}
$$

The D-factor is

$$
\mathrm{D}=\frac{4 \pi \rho_{0} r_{\mathrm{s}}^{3}}{D^{2}}\left[\log \left(\frac{D \theta}{2 r_{\mathrm{s}}}\right)+X\left(\frac{D \theta}{r_{\mathrm{s}}}\right)\right]
$$

with the limit of an infinite cusp $r_{\mathrm{s}} \rightarrow \infty$ regenerating the result of (11) with $\gamma=1$. In particular, as $\theta$ becomes large, the first term in Eq. (19) dominates and the D-factor diverges logarithmically. However, as $\theta \rightarrow 0$, the D-factor tends to

$$
\mathrm{D} \rightarrow 2 \pi \rho_{0} r_{\mathrm{s}} \theta^{2} \log \left(\frac{D \theta}{2 r_{\mathrm{s}}}\right)
$$

and so approaches zero quadratically.

\section{Comparisons}

Here, we compare our formulas with results in the literature. J-factors are often computed by finding the dark matter density profile that best fits the stellar kinematics through the spherical Jeans equations [10-12]. The multidimensional likelihood functions, which usually involve parameters controlling the dark halo structure as well as the velocity anisotropy, are then explored with Monte Carlo methods based on Bayesian parameter inference. An alternative method [13], which was used by the FermiLAT Collaboration, has some points of contact with our approach here. They use the empirical relationship (3) between velocity dispersion and mass enclosed within the half-light radius to circumvent solution of the Jeans equation. They then construct likelihood functions for each $\mathrm{dSph}$ from the luminosity, half-light radius and mass enclosed, together with priors, which are explored with a two-level Bayesian hierarchical model. Once the halo parameters are derived, the NFW profile is numerically integrated to find the J-factor.

The J-factors and D-factors computed using the full formula for a NFW cusp for a range of dSphs are given in Table I. Error bars are computed via propagation of errors in velocity dispersion, distance, and half-light radius using Eq. (16). We use an "ellipticity-corrected" version of the half-light radius which is given by the geometric average of the half-light radius as measured along the major and minor axes. This amounts to multiplying by $\sqrt{1-\epsilon}$ where $\epsilon$ is the ellipticity. Most data on half-light radii, distances, ellipticities and central velocity dispersions are extracted from the recent compilation of [32]. In addition, we use data from [17,33-37] on more recently discovered dwarfs. In cases where the ellipticity is only bounded we use the reported upper bound for our ellipticity-corrected half-light radius.
There is very little difference between values in this table and ones inferred from a Jeans analysis. We also show the $\mathrm{J}$-factors for an opening angle of $\theta=0.5^{\circ}$ against distance for the dSphs in Fig. 1. Naturally, the J-factors decrease with distance making the closest dSphs the most attractive candidates for dark matter annihilation detection. However, the diversity in measured velocity dispersions and half-light radii introduce variation into this $\mathrm{J}$-factor versus distance relation.

In Figs. 2 and 3, we show how our simple formulas compare to the results of Jeans calculations for the J-factor profiles for the classical and the ultrafaint dwarfs respectively. Red and yellow lines show the results obtained using Eq. (8). In each case, colored bands show the $1 \sigma$ range of values obtained by error propagation using the reported error on the data (half-light radius, line-of-sight velocity dispersion and heliocentric distance). ${ }^{1}$ The blue bands use Eq. (16) with $r_{\mathrm{s}}=5 R_{\mathrm{h}}$, which is a reasonable summary of results from phase-space modeling of the $\mathrm{dSphs}$, as given in Fig. 8 of Ref. [38]. For comparison, computed values for the J- and D-factors from [12] are also shown as solid circles with error bars. They are derived by assuming parametric laws for the light profile, the anisotropy of the stars and the dark halo profile. The latter is permitted to have a general double power-law structure with the density falling like $r^{-\gamma}$ at small radii and $r^{-\beta}$ at large radii. Solution of the spherical Jeans equations and subsequent projection then provides the line-of-sight velocity dispersions. By choosing priors on the unknown parameters and sampling the likelihood function through Markov chain Monte Carlo methods, Geringer-Sameth et al. [12] obtain constraints on the dark matter distribution. Hence, they can calculate the median value of the J-factors and the $1 \sigma$ distribution at selected angles (see their Table 2). The data points show the results of their calculations at two locations, namely $\theta_{1 / 2}$ or the angle containing 50 percent of the emission, and $\theta_{\max }=\arcsin \left(r_{\max }\right) / D$ where $r_{\max }$ is an estimate of the distance to the outermost member star with a measured radial velocity. We also show the J-factor profiles with $\pm 1 \sigma$ errors computed by Bonnivard et al. [39], which were obtained through a Jeans analysis similar to [12]. Finally, we show the constraints on the J-factors at $\theta=0.5^{\circ}$ from the Fermi-LAT Collaboration [40].

We see that the simple formula (8) performs reasonably well for the classical dwarfs, and better still for the ultrafaints. At larger $\theta$, the J-factor for pure power-law cusps is overestimated-here the assumption of an infinitely extended cusp breaks down. The full formula for the NFW halo (16) removes even this deficiency and reproduces the computational results extremely well. Note that it

\footnotetext{
${ }^{1}$ In the cases of Segue 2, Hydra I and Grus I only 95 percent, upper bounds, $C$, on the line-of-sight velocity dispersion are available. For these we assume a uniform distribution on the lineof-sight velocity dispersion between $0.1 \mathrm{~km} \mathrm{~s}^{-1}$ and $C / 0.95$.
} 
TABLE I. Annihilation and decay factors for dwarf spheroidals: we quote the predictions of the NFW formula from Eqs. (16) and (19) using $r_{s}=5 R_{\text {half }}$ at two angles - the angle between the center of the dwarf and an estimate of the distance to the outermost member star $\left(\theta_{\max }\right)$ and $\theta=0.5^{\circ}$. The dwarfs in the top section are classical dwarfs and those in the middle section are those ultrafaint dwarfs that have $J$ and $D$ estimates from the literature. The bottom section shows those ultrafaint dwarfs without preexisting $J$ or $D$ estimates. For these we adopt $\theta_{\max }=0.5^{\circ}$.

\begin{tabular}{|c|c|c|c|c|c|c|}
\hline Name & $\begin{array}{c}\text { Distance } \\
{[\mathrm{kpc}]}\end{array}$ & $\begin{array}{c}\theta_{\max } \\
{\left[{ }^{\circ}\right]}\end{array}$ & $\begin{array}{l}\log _{10} J\left(\theta_{\max }\right) \\
{\left[\mathrm{GeV}^{2} \mathrm{~cm}^{-5}\right]}\end{array}$ & $\begin{array}{l}\log _{10} J\left(0.5^{\circ}\right) \\
{\left[\mathrm{GeV}^{2} \mathrm{~cm}^{-5}\right]}\end{array}$ & $\begin{array}{c}\log _{10} D\left(\theta_{\max }\right) \\
{\left[\mathrm{GeV} \mathrm{cm} \mathrm{cm}^{-2}\right]}\end{array}$ & $\begin{array}{c}\log _{10} D\left(0.5^{\circ}\right) \\
{\left[\mathrm{GeV} \mathrm{cm} \mathrm{cm}^{-2}\right]}\end{array}$ \\
\hline Carina & $105 \pm 6$ & 1.26 & $18.03_{-0.34}^{+0.34}$ & $17.99_{-0.34}^{+0.34}$ & $18.37_{-0.17}^{+0.17}$ & $17.98_{-0.34}^{+0.34}$ \\
\hline Draco & $76 \pm 6$ & 1.3 & $18.92_{-0.25}^{+0.25}$ & $18.86_{-0.24}^{+0.24}$ & $18.82_{-0.12}^{+0.12}$ & $18.39_{-0.25}^{+0.25}$ \\
\hline Fornax & $147 \pm 12$ & 2.61 & $18.27_{-0.17}^{+0.17}$ & $18.15_{-0.16}^{+0.16}$ & $19.04_{-0.09}^{+0.09}$ & $18.26_{-0.17}^{+0.17}$ \\
\hline Leo I & $254 \pm 15$ & 0.45 & $17.80_{-0.28}^{+0.28}$ & $17.80_{-0.28}^{+0.28}$ & $17.84_{-0.14}^{+0.14}$ & $17.89_{-0.28}^{+0.28}$ \\
\hline Leo II & $233 \pm 14$ & 0.23 & $17.41_{-0.25}^{+0.25}$ & $17.44_{-0.25}^{+0.25}$ & $17.31_{-0.12}^{+0.12}$ & $17.62_{-0.25}^{+0.25}$ \\
\hline Sculptor & $86 \pm 6$ & 1.94 & $18.73_{-0.29}^{+0.29}$ & $18.65_{-0.29}^{+0.29}$ & $18.93_{-0.15}^{+0.15}$ & $18.33_{-0.29}^{+0.29}$ \\
\hline Sextans & $86 \pm 4$ & 1.7 & $18.04_{-0.29}^{+0.29}$ & $17.87_{-0.29}^{+0.29}$ & $18.76_{-0.15}^{+0.15}$ & $18.07_{-0.29}^{+0.29}$ \\
\hline Ursa Minor & $76 \pm 3$ & 1.37 & $19.18_{-0.24}^{+0.24}$ & $19.15_{-0.24}^{+0.25}$ & $18.84_{-0.12}^{+0.12}$ & $18.45_{-0.24}^{+0.24}$ \\
\hline Boötes I & $66 \pm 2$ & 0.47 & $16.64_{-0.38}^{+0.64}$ & $16.65_{-0.38}^{+0.64}$ & $17.25_{-0.19}^{+0.32}$ & $17.28_{-0.38}^{+0.64}$ \\
\hline Coma Berenices & $44 \pm 4$ & 0.31 & $18.64_{-0.32}^{+0.32}$ & $18.67_{-0.32}^{+0.33}$ & $17.83_{-0.16}^{+0.16}$ & $18.06_{-0.32}^{+0.32}$ \\
\hline Canes Venatici I & $218 \pm 10$ & 0.53 & $17.27_{-0.11}^{+0.11}$ & $17.27_{-0.11}^{+0.11}$ & $17.81_{-0.05}^{+0.05}$ & $17.78_{-0.11}^{+0.11}$ \\
\hline Canes Venatici II & $160 \pm 4$ & 0.13 & $17.63_{-0.40}^{+0.40}$ & $17.65_{-0.40}^{+0.40}$ & $16.91_{-0.20}^{+0.20}$ & $17.37_{-0.40}^{+0.40}$ \\
\hline Hercules & $133 \pm 12$ & 0.28 & $16.79_{-0.45}^{+0.45}$ & $16.83_{-0.45}^{+0.45}$ & $17.10_{-0.22}^{+0.22}$ & $17.38_{-0.45}^{+0.45}$ \\
\hline Leo IV & $154 \pm 6$ & 0.16 & $16.56_{-0.90}^{+0.90}$ & $16.64_{-0.90}^{+0.90}$ & $16.68_{-0.45}^{+0.45}$ & $17.22_{-0.90}^{+0.90}$ \\
\hline Leo V & $178 \pm 10$ & 0.07 & $16.82_{-0.70}^{+1.05}$ & $16.94_{-0.72}^{+1.05}$ & $16.35_{-0.35}^{+0.53}$ & $17.23_{-0.70}^{+1.05}$ \\
\hline Leo $\mathrm{T}$ & $417 \pm 19$ & 0.08 & $17.28_{-0.37}^{+0.37}$ & $17.32_{-0.37}^{+0.38}$ & $16.70_{-0.19}^{+0.19}$ & $17.35_{-0.38}^{+0.38}$ \\
\hline Segue 1 & $23 \pm 2$ & 0.35 & $19.39_{-0.39}^{+0.39}$ & $19.41_{-0.40}^{+0.39}$ & $18.03_{-0.20}^{+0.20}$ & $18.17_{-0.39}^{+0.39}$ \\
\hline Segue 2 & $35 \pm 2$ & 0.19 & $17.06_{-1.75}^{+0.86}$ & $17.11_{-1.76}^{+0.85}$ & $16.64_{-0.87}^{+0.43}$ & $17.08_{-1.75}^{+0.86}$ \\
\hline Ursa Major I & $97 \pm 4$ & 0.43 & $18.47_{-0.25}^{+0.25}$ & $18.48_{-0.25}^{+0.25}$ & $18.08_{-0.13}^{+0.13}$ & $18.15_{-0.25}^{+0.25}$ \\
\hline Ursa Major II & $32 \pm 4$ & 0.53 & $19.38_{-0.39}^{+0.39}$ & $19.38_{-0.39}^{+0.39}$ & $18.51_{-0.19}^{+0.19}$ & $18.48_{-0.39}^{+0.39}$ \\
\hline Willman 1 & $38 \pm 7$ & 0.5 & $19.29_{-0.62}^{+0.91}$ & $19.29_{-0.62}^{+0.91}$ & $18.03_{-0.32}^{+0.45}$ & $18.03_{-0.62}^{+0.91}$ \\
\hline Reticulum II & $30 \pm 3$ & 1.0 & $18.72_{-0.32}^{+0.85}$ & $18.71_{-0.32}^{+0.84}$ & $18.19_{-0.17}^{+0.42}$ & $17.93_{-0.32}^{+0.85}$ \\
\hline Tucana II & $57 \pm 5$ & 1.0 & $19.10_{-0.58}^{+0.88}$ & $19.05_{-0.58}^{+0.87}$ & $18.79_{-0.29}^{+0.44}$ & $18.45_{-0.58}^{+0.88}$ \\
\hline Horologium I & $79 \pm 7$ & 0.5 & $18.64_{-0.39}^{+0.95}$ & $\ldots$ & $17.78_{-0.20}^{+0.47}$ & $\ldots$ \\
\hline Hydra II & $134 \pm 10$ & 0.5 & $16.56_{-1.85}^{+0.87}$ & $\cdots$ & $16.89_{-0.92}^{+0.44}$ & $\cdots$ \\
\hline Pisces II & $182 \pm 18$ & 0.5 & $17.90_{-0.80}^{+1.14}$ & $\cdots$ & $17.41_{-0.40}^{+0.57}$ & $\cdots$ \\
\hline Grus I & $120 \pm 11$ & 0.5 & $17.96_{-1.93}^{+0.90}$ & $\cdots$ & $17.59_{-0.96}^{+0.46}$ & $\cdots$ \\
\hline
\end{tabular}

is now just as straightforward to work out the entire profile as to compute a single value. In principle, the full profile may give information on the structure of the dark halo if a $\mathrm{dSph}$ is detected in $\gamma$ rays. The largest discrepancy between our calculation and that of Geringer-Sameth et al. [12] is for Boötes I. This may arise because Boötes I possesses both a hot and cold component [42], whereas our calculation uses the velocity dispersion of the colder population only. Another possible source of this discrepancy is that the best-fitting dark halo density law inferred by [12] may deviate from the strict NFW form-in fact, they report that Boötes I has a median value of its inner slope $\gamma$ of 0.53 and outer slope $\beta$ of 5.9. This discrepancy persists when comparing against the results of Bonnivard et al. [39]. The other striking anomaly is when comparing our result for Segue 1 with Bonnivard et al. [39]. This is caused by different ways of designating a subset of the spectroscopically observed stars as "members" of Segue 1 as opposed to Milky Way foreground contaminants, as originally noted in [43]. Bonnivard, Maurin, and Walker [41] have shown that the determination of Segue 1's J-factor (and, implicitly, its velocity dispersion) is extremely sensitive to the inclusion or exclusion of a small number of stars with ambiguous membership status. The J-factors computed in [39] are 


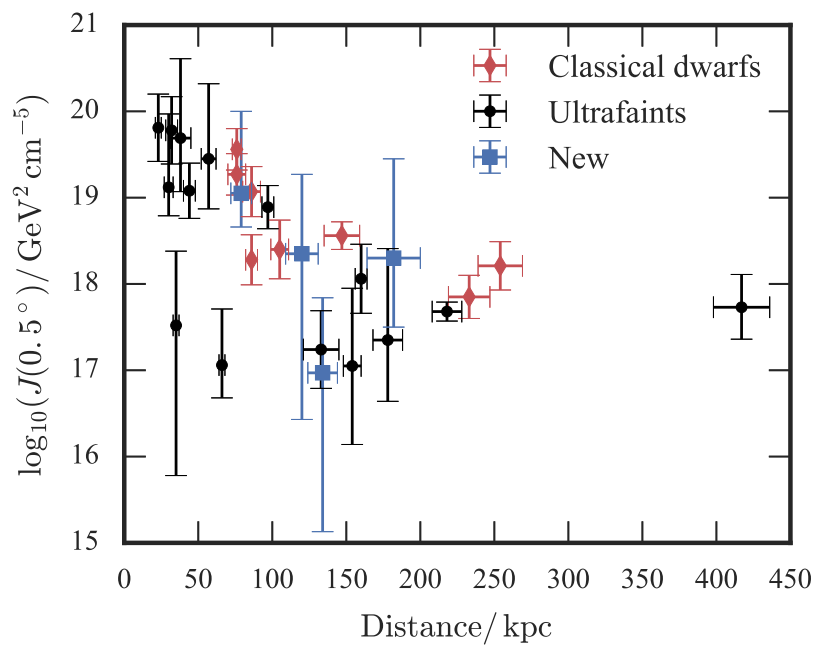

FIG. 1. Dwarf annihilation factors against distance: the points show the $\mathrm{J}$-factors computed for an opening angle of $0.5^{\circ}$ using the simple NFW formula presented in this paper with $r_{s}=5 R_{\mathrm{h}}$. The red diamonds correspond to the classical dwarfs, the black circles to the ultrafaints, and the blue squares to four recently discovered dwarfs with no preexisting literature estimates of their J-factors. based on more stringent membership criteria for Segue 1 as compared to the analyses of [12] and [40].

A straightforward conclusion from Figs. 2 and 3 is that Draco, Ursa Minor, Sculptor, Coma Berenices, Reticulum II, Tucana II and Ursa Major II are the most favourable dSphs for which to look for signatures of dark matter annihilation. Willman 1 also has a high J-factor but the assumption of dynamical equilibrium for this object is dubious as it appears to be severely tidally disrupted [44]. Similarly, Segue 1 has a high J-factor but the issue of foreground contamination of the spectroscopic sample brings this into doubt $[41,43]$.

Figure 4 gives predictions for 4 recently discovered ultrafaints for which there are no J-factors in the literature. These objects are Horologium I and Grus I (discovered in Dark Energy Survey Data [17,45]), Hydra II (discovered by the Survey of the Magellanic Stellar History) and [34], Pisces II (discovered in Sloan Digital Sky Survey data [35]). Keck/DEIMOS spectroscopy of Hydra II and Pisces II has recently been published [36]. The Gaia-ESO survey has measured the velocities of 5 stars in Horologium I using the VLT/Giraffe combination [46], while Magellan/M2FS has been used to target 7 stars in Grus I [37]. Using the data in these papers, we calculate the J-factors. The uncertainties are calculated by Monte Carlo sampling and then estimating the

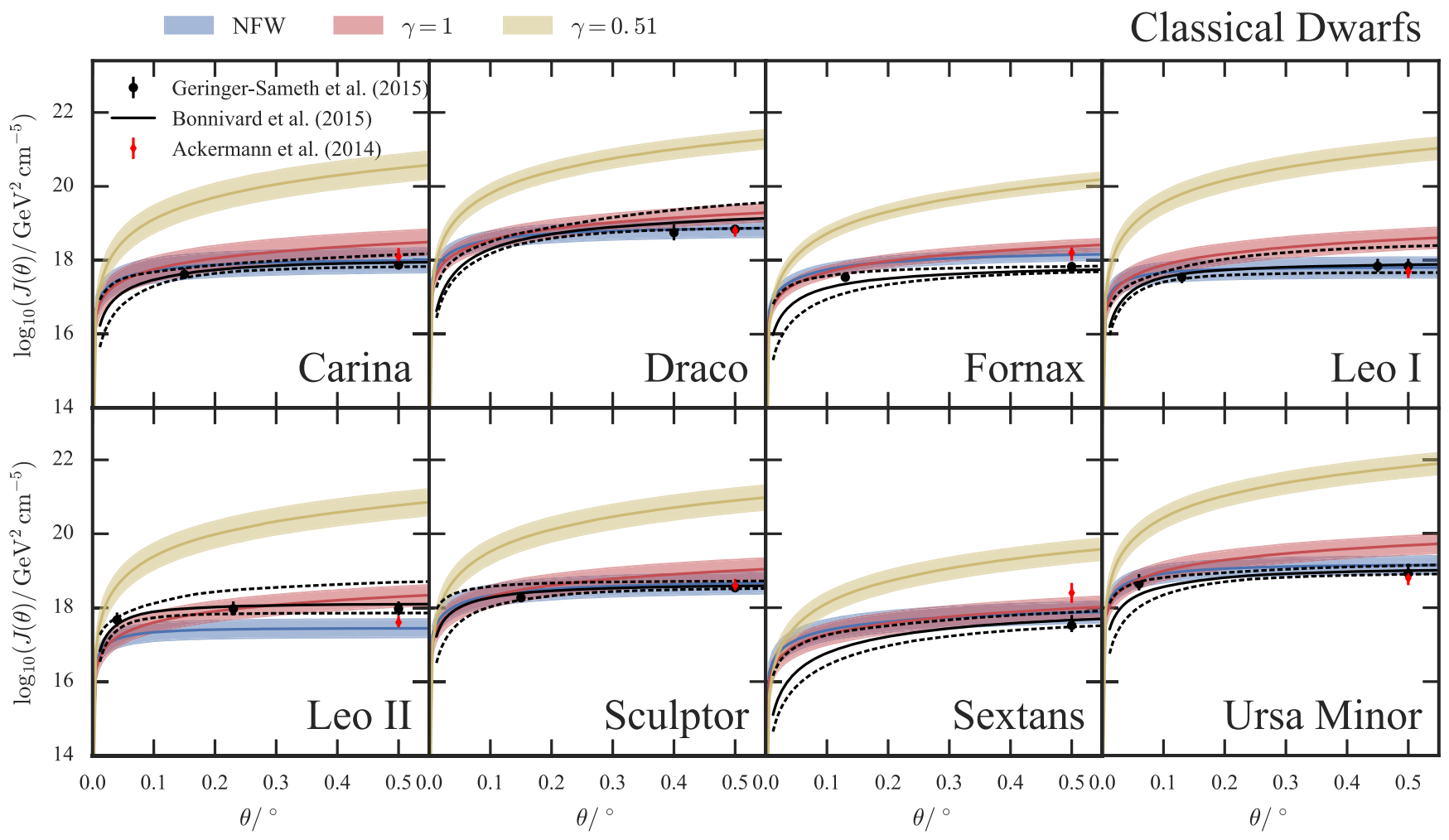

FIG. 2. Classical dwarf annihilation factors: the points are taken from the full Jeans analysis of [12]. The red and yellow bands are the estimates from Eq. (8) using $\gamma=1$ and $\gamma=0.51$ respectively. The blue band is the estimate from Eq. (16) using $r_{s}=5 R_{\text {half }}$. The median and $\pm 1 \sigma$ estimates of $\log _{10}(J(\theta))$ from [39] are given by the black solid and dashed lines respectively. The red diamonds are the estimates of $\log _{10}\left(J\left(0.5^{\circ}\right)\right)$ from [40]. 


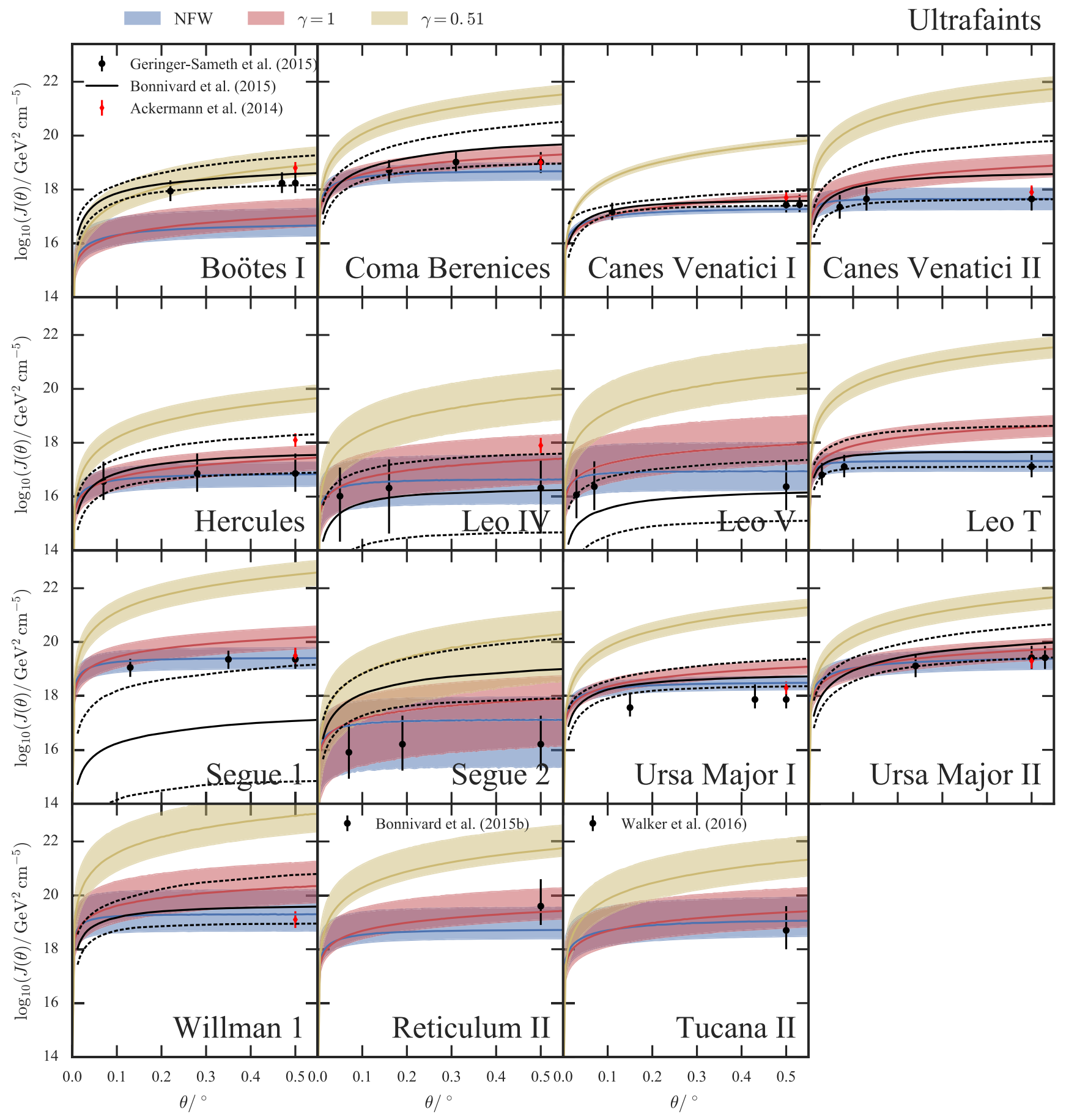

FIG. 3. Ultrafaint dwarf annihilation factors: see caption of Fig. 2. The points for Tucana II and Reticulum II are taken from Refs. [37] and [41] respectively.

$1 \sigma$ bound on the J-factor from the ensuing distributions. It is clear that Horologium $\mathrm{I}$ is another excellent candidate with a J-factor comparable to Reticulum II and Tucana II.

We show in Figs 5-7 analogous plots for the D-factor for classical dwarfs, ultrafaints and predictions for recent discoveries. Both the simple formula (11) and the exact result for an NFW cusp (19) do an excellent job of reproducing the results in the literature-with much greater economy of effort.

\section{APPLICATIONS}

\section{A. Cusps and cores}

So far, we have focussed on the NFW model with its $1 / r$ density cusp. Although this has a preferred status because 


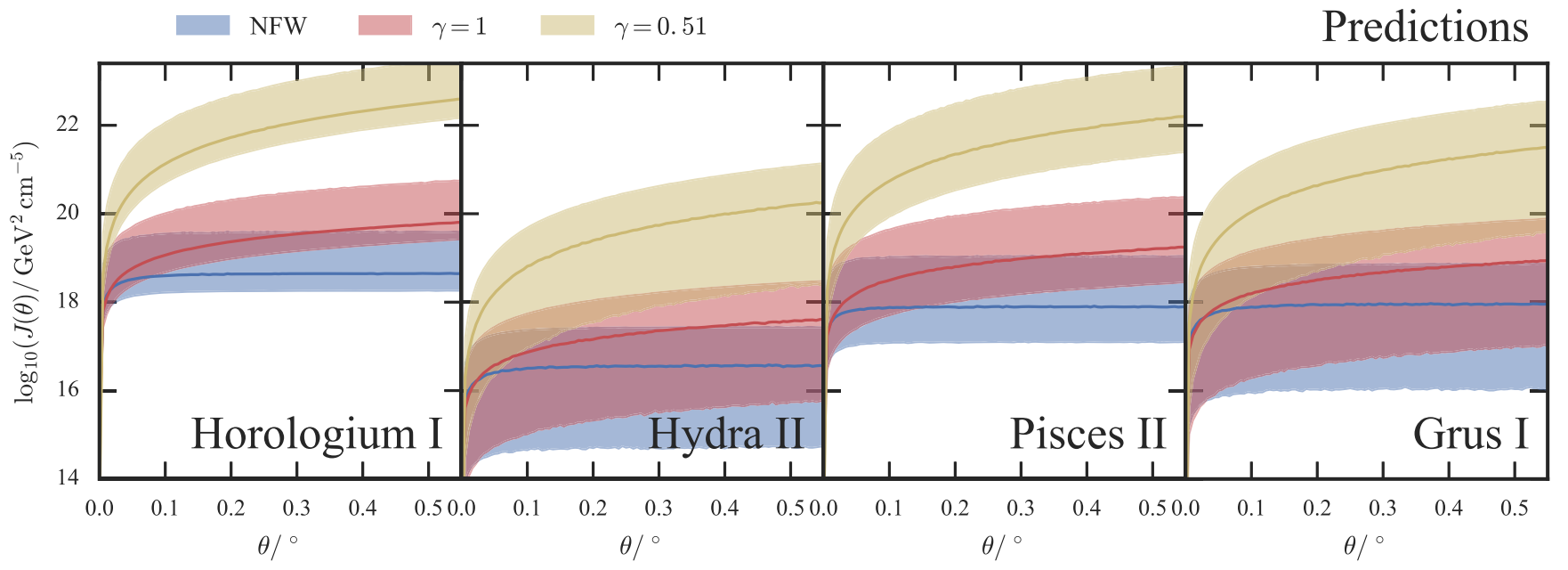

FIG. 4. Predictions for ultrafaint dwarf annihilation factors: see caption of Fig. 2.

of its importance in theories of galaxy formation, there is some evidence that dark haloes may have a different structure. For example, many of the detailed kinematical fits reported by [12] have milder cusps, often with $\rho_{\mathrm{DM}} \sim$ $r^{-1 / 2}$ at small radii. There is also strong observational evidence that some of the dwarf galaxies are mildly cusped or even cored. This includes the persistence of substructure in Ursa Minor [47], the survival of globular clusters in Fornax [48], and the kinematics of multiple populations $[25,28,49,50]$. Here, somewhat speculatively, we suppose that the J-profile can be mapped out as a function of $\theta$ and ask what can then be deduced about the dark halo structure.

A flexible set of dark halos with double power-law structure has the form $[9,51]$

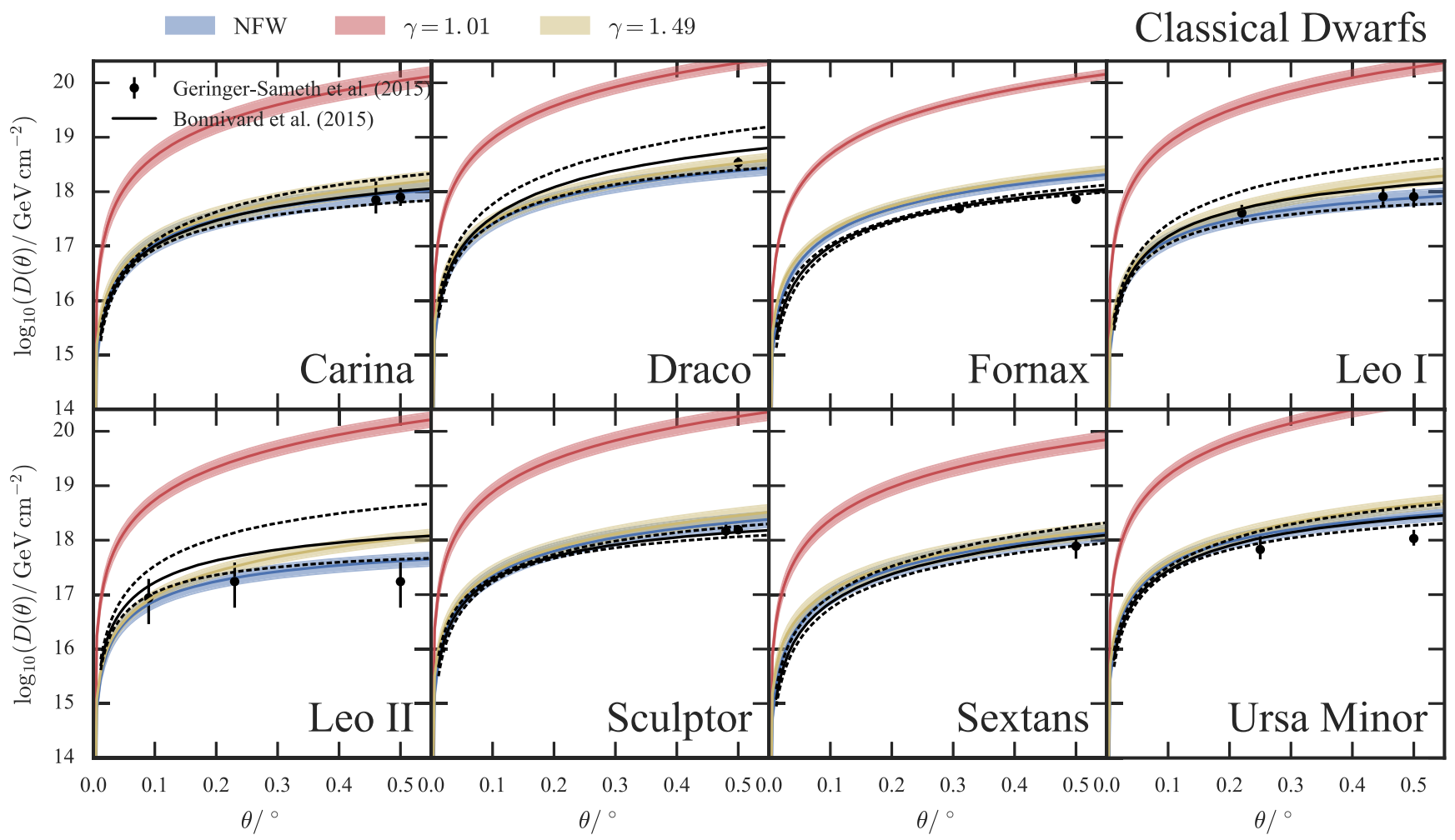

FIG. 5. Classical dwarfs decay factors: the points are taken from the full Jeans analysis of [12]. The red and yellow bands are the estimates from Eq. (11) using $\gamma=1$ and $\gamma=1.49$ respectively. The blue band is the estimate from Eq. (19) using $r_{s}=5 R_{\text {half }}$. The median and $\pm 1 \sigma$ estimates of $\log _{10}(D(\theta))$ from [39] are given by the black solid and dashed lines respectively. 


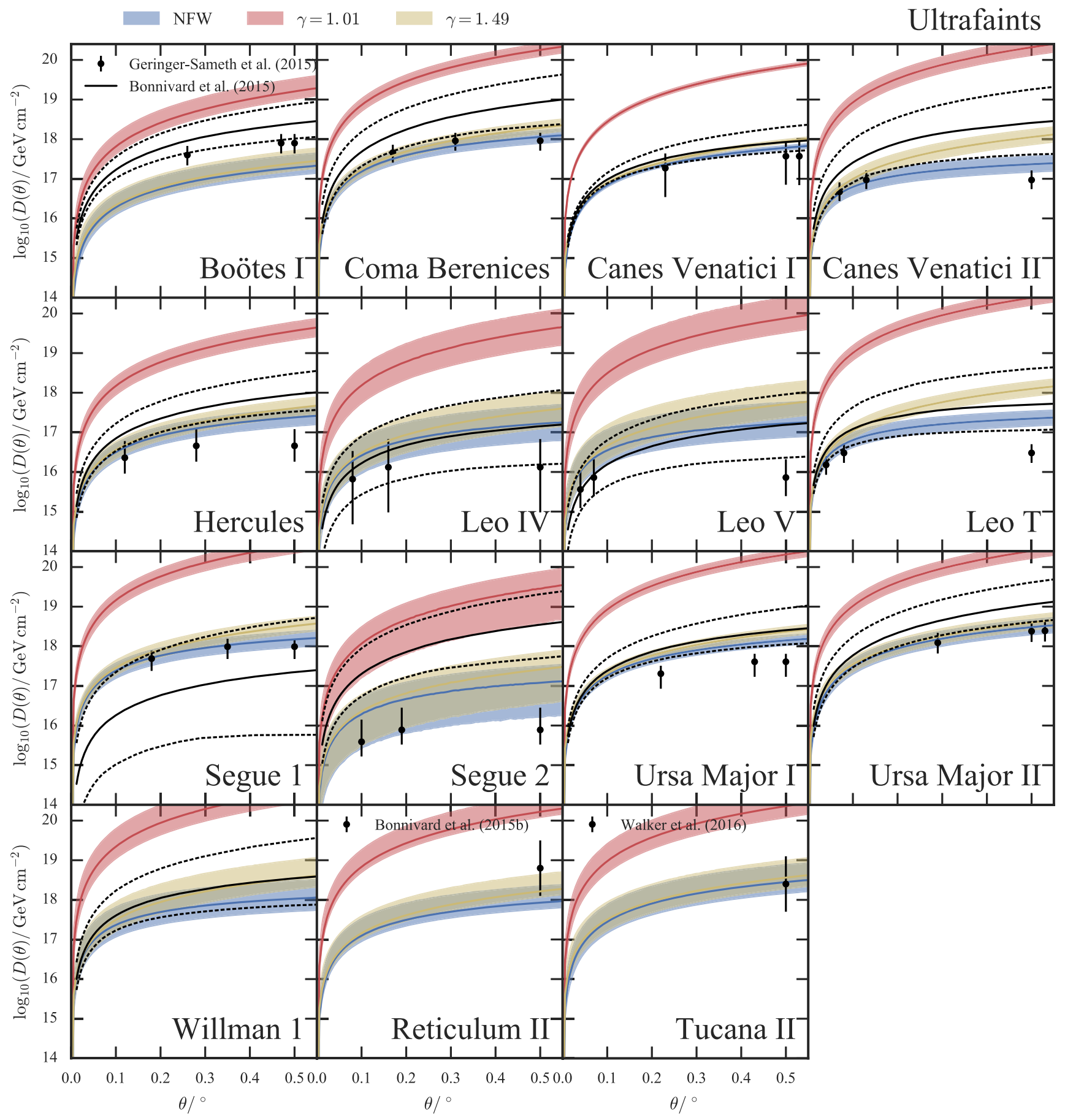

FIG. 6. Ultrafaint dwarfs decay factors: see caption of Fig. 5.

$$
\rho_{\mathrm{DM}}(r)=\rho_{0}\left(\frac{r}{r_{\mathrm{s}}}\right)^{-\gamma}\left(1+\left(\frac{r}{r_{\mathrm{s}}}\right)^{\alpha}\right)^{(\gamma-\beta) / \alpha}
$$

with $\alpha, \beta$ and $\gamma$ as positive numbers. The familiar NFW profile is recovered in the case $(\theta, \beta, \gamma)=(1,3,1)$. Although the J-factors for this entire class of models are not analytic, nonetheless we can easily work out their general properties. At small radii, the density is cusped like $r^{-\gamma}$. For strong cusps with $1 / 2<\gamma<3 / 2$, the behavior can be deduced from our work on infinite cusps in Sec. II by making the identification $\rho_{0} r_{s}^{\gamma}=(3-\gamma) \sigma_{\text {los }}^{2} /\left(\pi R_{\mathrm{h}}^{2-\gamma} G\right)$. We see from Eq. (8) that the J-factor increases like $J \propto \theta^{3-2 \gamma}$ on moving outwards from the center of the halo. For weaker cusps and cores $(0 \leq \gamma<1 / 2)$, the dominant term in the J-factor near the origin is always a gentler quadratic, namely $J \propto \theta^{2}$, as the $\gamma$-ray emission is now no 


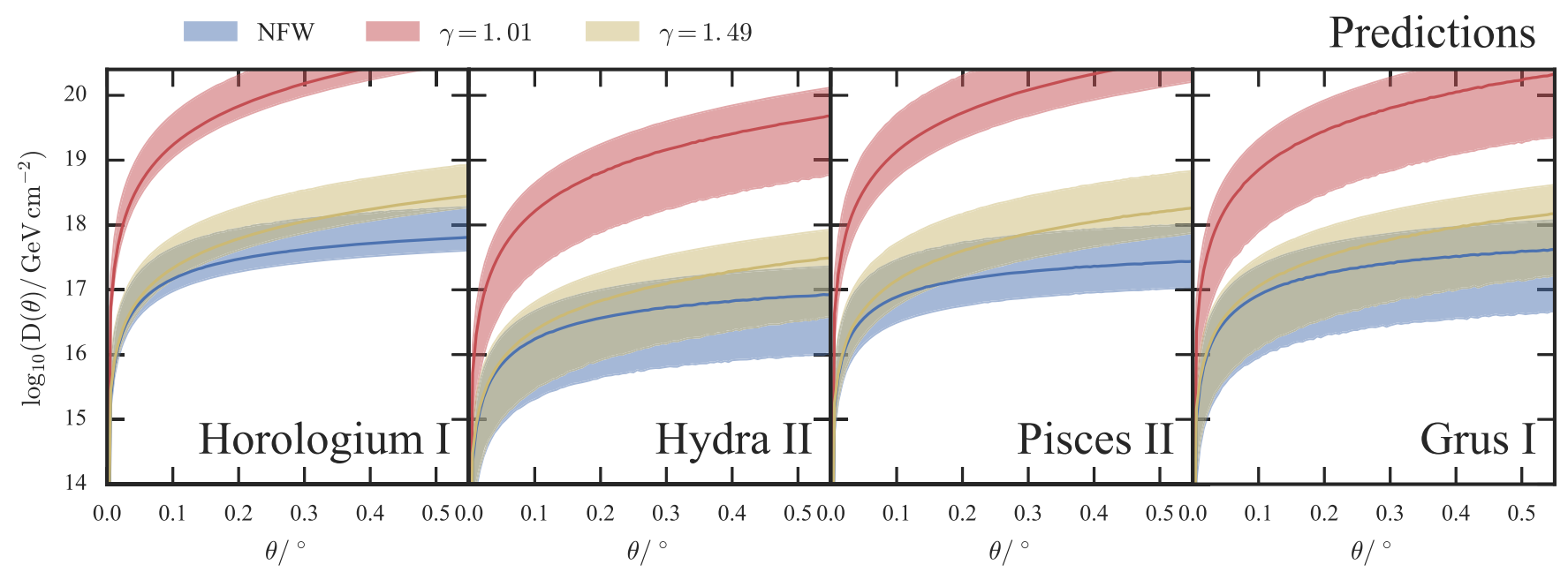

FIG. 7. Predictions for ultrafaint dwarf decay factors: see caption of Fig. 5.

longer dominated by the very center. Beyond the scale radius $r_{\mathrm{s}}$, the $\mathrm{J}$-factor turns over and tends to a constant value. This is given by

$$
\mathrm{J} \rightarrow \frac{4 \pi r_{\mathrm{s}}^{3} \rho_{0}^{2}}{D^{2}} \frac{\Gamma[(2 \beta-3) / \alpha] \Gamma[(3-2 \gamma) / \alpha]}{\alpha \Gamma[2(\beta-\gamma) / \alpha]}
$$

Formally, we require $\beta>3 / 2$ and $\gamma<3 / 2$ for convergence, though this is satisfied by almost all astrophysically realistic models. Notice when $(\alpha, \beta, \gamma)=(1,3,1)$, we recover the asymptotic value for the J-factor of a NFW halo [Eq. (18)].

The behavior of the D-factor can be worked out in a similar way, though now the boundary between strong and weak cusps is at $\gamma=1$. For cusps with $\gamma<1$, the D-factor initially rises like $J \propto \theta^{3-\gamma}$, while for cusps with $\gamma \leq 1$, the rise is quadratic $J \propto \theta^{2}$. At large angles, the $\mathrm{D}$-factor turns over to the constant value

$$
\mathrm{D} \rightarrow \frac{4 \pi r_{\mathrm{s}}^{3} \rho_{0}}{D^{2}} \frac{\Gamma[(\beta-3) / \alpha] \Gamma[(3-\gamma) / \alpha]}{\alpha \Gamma[(\beta-\gamma) / \alpha]}
$$

provide $\beta>3$ and $\gamma<3$, as required for convergence. For the NFW model, $\beta=3$ and so the D-factor logarithmically diverges.

We can illustrate this behavior with two simple models. A prototype of a weakly cusped dark halo is the model with $(\alpha, \beta, \gamma)=(1,3,1 / 2)$, namely,

$$
\rho(r)=\frac{\rho_{0} r_{\mathrm{s}}^{3}}{r^{1 / 2}\left(r+r_{\mathrm{s}}\right)^{5 / 2}}
$$

for which the J-factor is

$$
\begin{aligned}
\mathrm{J}= & \frac{\pi \rho_{0}^{2}}{6 \Delta^{6} r_{\mathrm{s}}^{3}}\left[6 D^{2} r_{\mathrm{s}}^{2} \theta^{2}-2 D^{4} \theta^{4}-19 r_{\mathrm{s}}^{4}\right. \\
& \left.+3 r_{\mathrm{s}}^{2}\left(4 r_{\mathrm{s}}^{2}+D^{2} \theta^{2}\right) X\left(\frac{D \theta}{r_{\mathrm{s}}}\right)\right] .
\end{aligned}
$$

Its asymptotic value is

$$
\mathrm{J} \rightarrow \frac{\pi r_{\mathrm{s}}^{3} \rho_{0}^{2}}{3 D^{2}}
$$

while its central value is

$$
\mathrm{J} \rightarrow \frac{\pi r_{\mathrm{s}} \rho_{0}^{2}}{6}\left(6 \log \left(\frac{4 r_{\mathrm{s}}^{2}}{D^{2} \theta^{2}}\right)-19\right) \theta^{2}
$$

The D-factor is not analytic, but diverges logarithmically at large radii.

A prototype for cored dark haloes is the famous Plummer (1911) model, which corresponds to $(\alpha, \beta, \gamma)=(2,5,0)$. This is often used for modeling cored dark haloes (as well as clusters and the stellar populations in dSphs). The density is

$$
\rho(r)=\frac{\rho_{0} r_{\mathrm{s}}^{5}}{\left(r^{2}+r_{\mathrm{s}}^{2}\right)^{5 / 2}}
$$

The $\mathrm{J}$-factor is

$$
\mathrm{J}=\frac{5 \pi^{2}}{64} \frac{\rho_{0}^{2} r_{\mathrm{s}}^{3}}{D^{2}}\left[1-\frac{r_{\mathrm{s}}^{7}}{\left(r_{\mathrm{s}}^{2}+D^{2} \theta^{2}\right)^{\frac{7}{2}}}\right]
$$

Notice that the J-factor behaves like $\theta^{2}$ at small angles, whereas the asymptotic value is in accord with our general rule (22). The D-factor is also very simple 


$$
\mathrm{D}=\frac{4 \pi}{3} \frac{\rho_{0} r_{\mathrm{s}}^{3} \theta^{2}}{\left(r_{\mathrm{s}}^{2}+D^{2} \theta^{2}\right)}
$$

It again approaches zero quadratically, and tends to the asymptotic value given by (23). This is in accord with our general results.

In principle, if the variation of the J-factor can be mapped out with integration angle, then valuable information on the structure of the dark halo can be gleaned. The behavior at small angles can yield information on the cusp slope $\gamma$. In particular, if $\gamma>1 / 2$, then the logarithmic gradient of $J$ with respect to $\theta$ is the cusp slope. Alternatively, if $J \propto \theta^{2}$, then the cusp is either very mild or the dark halo is cored. Similarly, the asymptotic value of the J-factor is controlled by the normalisation $\rho_{0}$, scale-length $r_{\mathrm{s}}$ and the density falloff $\beta$. Some of these quantities, such as $r_{\mathrm{s}}$ can of course be constrained by the stellar kinematics of the stars, so the asymptotic value may enable a complete solution for the halo to be obtained.

\section{B. The sweet spot}

The idea that there is a sweet spot-a location in which the measured quantity is reasonably robust against changes in the underlying dark halo model-has proved quite powerful in studies of dSphs. Of course, the most successful instance is the hypothesis that the mass of dark matter enclosed within the half-light radius of the stellar population is reasonably robust against changes in anisotropy $[26,27]$. Similar ideas have been used to identify radii at which the enclosed mass is insensitive to changes in the underlying halo models $[38,52]$. It is therefore natural to wonder if there is a special angle $\theta$ at which the J-factor is particularly robust.

Walker et al. [10] noticed from their Jeans solutions for dark matter haloes for the Carina dSph that the J-factor was least sensitive to changes in the inner slope $\gamma$, outer slope $\beta$ and velocity anisotropy at an integration angle $\theta \approx 2 R_{\mathrm{h}} / D$. They then generated mock data sets from distribution functions for models with varying inner slope $\gamma$ and velocity anisotropy, but fixed outer slope $\beta$, which supported the idea of a sweet spot at $\theta \approx 2 R_{\mathrm{h}} / D$.

We test this directly in Fig. 8 by plotting the range of $\mathrm{J}$-factors and $\mathrm{D}$-factors of $(\alpha, \beta, \gamma)$ models with the same mass enclosed within the half-light radius of the stars $R_{\mathrm{h}}$. This mass was chosen using the velocity dispersion and half-light radius of Reticulum II. The range of models explored were $\alpha \in[1 ., 2],. \quad \beta \in[3 ., 6],. \quad \gamma \in[0 ., 1.2]$. Although we have not explicitly used any kinematic data, it is nonetheless encoded in the models using the fact that the mass enclosed with $R_{\mathrm{h}}$-and hence the luminosity weighted velocity dispersion-is the same for all the models [26,27]. The vertical line in Fig. 8 shows that the integration angle $\theta \approx R_{\mathrm{h}} / D$ at which the scatter in the $\mathrm{J}$-factors of the models is minimized. Notice even though the mass enclosed within $R_{\mathrm{h}}$ is exactly the same for all the

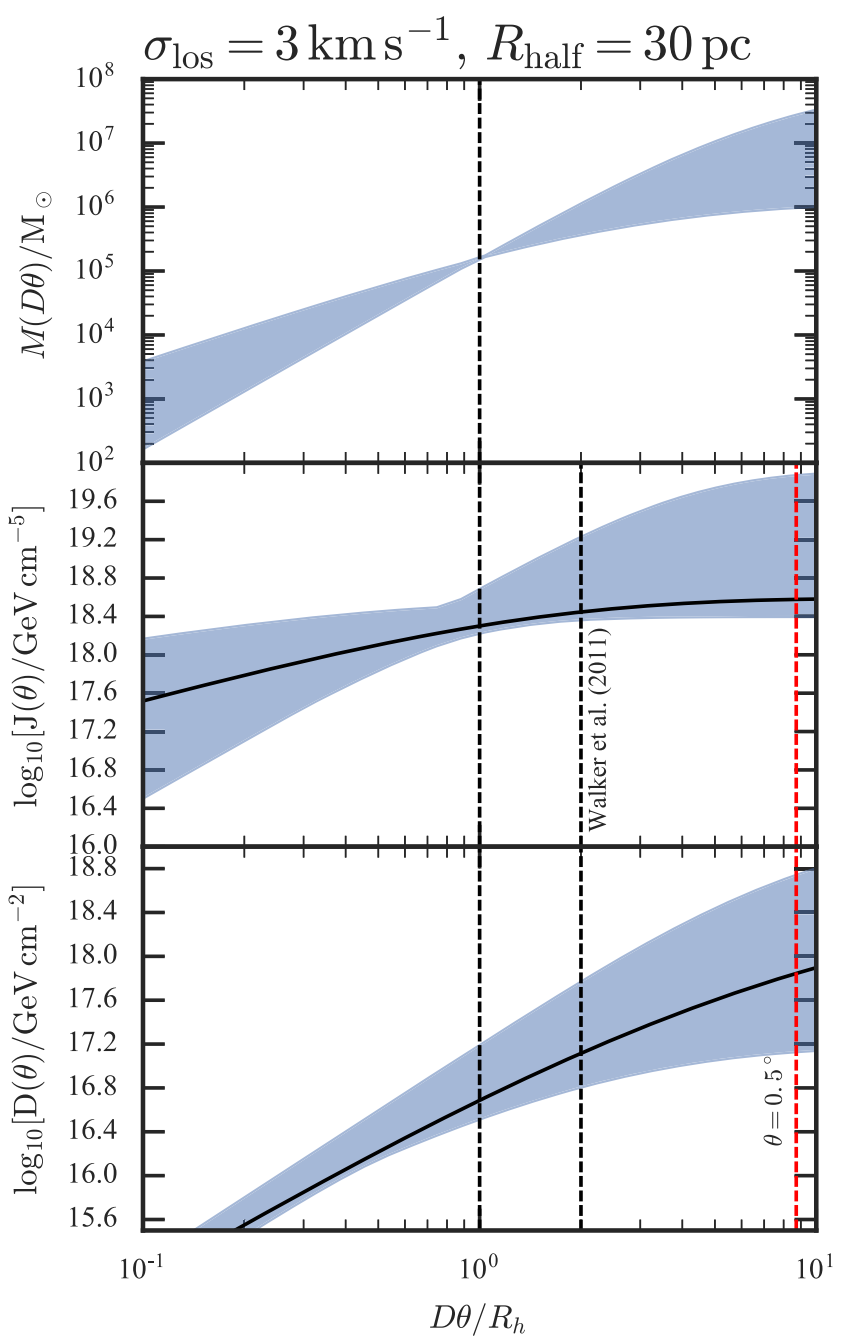

FIG. 8. Comparison of J-factors and D-factors of $(\alpha, \beta, \gamma)$ dark halos with the same mass enclosed within the half-light radius $\left(R_{\mathrm{h}}=30 \mathrm{pc}\right)$, represented by the left black dashed line. The parameters chosen approximately match those of Reticulum II and we set $r_{s}=5 R_{\mathrm{h}}$. The blue band shows the range of possible profiles when $\alpha \in[1 ., 2],. \beta \in[3 ., 6],. \gamma \in[0 ., 1.2]$. The solid black line corresponds to the NFW profile. Notice that the $\mathrm{J}$-factors of these models show least scatter at the angle $\theta \approx R_{\mathrm{h}} / D$. The radii suggested in [10] is indicated by a vertical dotted line, and-at least for these models-shows greater scatter. The D-factors show the smallest scatter at a much smaller angle. The red dashed vertical line shows Fermi-LAT resolution for Reticulum II.

models, there is nonetheless some scatter in the J-factors even at $\theta=R_{\mathrm{h}} / D$. Also shown with a vertical line is the angle $\theta \approx 2 R_{\mathrm{h}} / D$ suggested by [10], at which the scatter is rather greater.

Presumably, the explanation of this discrepancy is that the models constructed in [10], which are derived from Markov chain Monte Carlo fits to discrete radial velocities, do not exactly satisfy the empirical relation on mass enclosed within the half-light radius and velocity dispersion. Of course, the actual integration angle used 
is a tradeoff between gathering power and minimizing the uncertainty in the dark halo properties.

\section{CONCLUSIONS}

The main advance in this paper is the provision of very simple formulas for $\mathrm{J}$-factors for indirect dark matter detection. This includes power-law spherical cusps, as well as the exact result for the Navarro-Frenk-White [22] model. These compact formula offer significant savings in effort over Jeans solution methods driven by Markov Chain Monte Carlo engines. Especially for the ultrafaint dwarfs, for which the size of the datasets of discrete velocities is tiny, this provides an entirely elementary method of evaluating J-factors. The exact formulas for the J-factors and D-factors of the NFW should prove particularly useful, as there are reasons increasing in quality and quantity (e.g., [20,21,23,24]) for believing that many of the ultrafaints have pristine dark matter haloes, even if some of the largest dSphs most probably have cored haloes (e.g., $[25,28,47,49])$.

We have computed the J-factors (and $1 \sigma$ uncertainties) for all the known dSph galaxies with kinematic data. This includes the first estimates for Horologium I and Grus I-recently discovered in Dark Energy Survey Data data-as well as Hydra II and Pisces II. From this compendium, the ultrafaints with the highest J-factors are Ursa Major II, Reticulum II, Tucana II and Horologium I. They are very attractive targets for indirect detection experiments. Although our formulas assign Willman 1 and Segue 1 high J-factors, these ambiguous objects may be extended clusters suffering disruption in the Galactic tidal field rather than dwarf galaxies, perhaps complicating or even invalidating previous estimates of $\sigma_{\text {los }}$ for these systems. Amongst the classical dSphs, Draco, Sculptor and Ursa Minor have the highest J-factors.

If the J-factor profile can be mapped out as a function of integration angle, then this raises the possibility of inferring properties for the dark matter halo directly. We have shown for general halo models that the J-factor rises from the origin, yet approaches a constant asymptotic value with increasing integration angle. The behavior at the origin encodes information on the inner cusp slope $\gamma$. If the model is cored or weakly cusped $(0 \leq \gamma \leq 1 / 2)$, then the J-factor rises quadratically from the origin, $J \propto \theta^{2}$. If the model is more strongly cusped $(1 / 2<\gamma<3 / 2)$, then $J \propto \theta^{3-2 \gamma}$. The asymptotic value of the J-factor-which can be worked out analytically-depends on both inner slope $\gamma$, outer slope $\beta$ as well as the halo scale length and normalization.

Finally, we have identified a sweet spot, or preferential integration angle, at which the J-factor is robust against changes in the dark halo model. This is at the integration angle $\theta=R_{\mathrm{h}} / D$, or the ratio of the $\mathrm{dSphs}$ half-light radius $R_{\mathrm{h}}$ to distance from the observer $D$. This result holds good for models that exactly obey the empirical relation between velocity dispersion and mass enclosed within half-light radius $[26,27]$.

An important outstanding problem is the extension of this work to flattened geometries. Most dSphs are flattened-and some of the most promising targets such as Reticulum II or Horologium I are very highly flattened. Spherical models can provide useful guides, especially for the largest classical dSphs (like Leo I or Fornax) that look nearly round on the sky. They are least useful for the flattened ultrafaints. In a companion paper, we show how to extend the techniques presented here to explore flattened and triaxial geometries [19].

\section{ACKNOWLEDGMENTS}

The authors thank the anonymous referees for helpful reports, as well as Vasily Belokurov and Matt Walker for useful discussions. J. L. S. acknowledges financial support from the Science and Technology Facilities Council (STFC) of the United Kingdom.
[1] J. Kleyna, M. I. Wilkinson, N. W. Evans, G. Gilmore, and C. Frayn, Mon. Not. R. Astron. Soc. 330, 792 (2002).

[2] M. L. Mateo, Annu. Rev. Astron. Astrophys. 36, 435 (1998).

[3] S. Colafrancesco, S. Profumo, and P. Ullio, Phys. Rev. D 75, 023513 (2007).

[4] T. L. Culverhouse, N. W. Evans, and S. Colafrancesco, Mon. Not. R. Astron. Soc. 368, 659 (2006).

[5] N. W. Evans, F. Ferrer, and S. Sarkar, Phys. Rev. D 69, 123501 (2004).

[6] G. Lake, Nature (London) 346, 39 (1990).

[7] L. Bergström, P. Ullio, and J. H. Buckley, Astropart. Phys. 9, 137 (1998).
[8] A. A. Abdo, M. Ackermann, M. Ajello et al., Astrophys. J. 712, 147 (2010).

[9] A. Charbonnier, C. Combet, M. Daniel et al., Mon. Not. R. Astron. Soc. 418, 1526 (2011).

[10] M. G. Walker, C. Combet, J. A. Hinton, D. Maurin, and M. I. Wilkinson, Astrophys. J. Lett. 733, L46 (2011).

[11] V. Bonnivard, C. Combet, D. Maurin, and M. G. Walker, Mon. Not. R. Astron. Soc. 446, 3002 (2015).

[12] A. Geringer-Sameth, S. M. Koushiappas, and M. Walker, Astrophys. J. 801, 74 (2015).

[13] G. D. Martinez, Mon. Not. R. Astron. Soc. 451, 2524 (2015). 
[14] D. B. Zucker, V. Belokurov, N. W. Evans et al., Astrophys. J. Lett. 650, L41 (2006).

[15] A. J. Deason, V. Belokurov, N. W. Evans, L. L. Watkins, and M. Fellhauer, Mon. Not. R. Astron. Soc. 425, L101 (2012).

[16] N. G. Martin, J. T. A. de Jong, and H.-W. Rix, Astrophys. J. 684, 1075 (2008).

[17] S. E. Koposov, V. Belokurov, G. Torrealba, and N. W. Evans, Astrophys. J. 805, 130 (2015).

[18] C. F. P. Laporte, M. G. Walker, and J. Peñarrubia, Mon. Not. R. Astron. Soc. 433, L54 (2013).

[19] J. L. Sanders, N. W. Evans, A. Geringer-Sameth, and W. Dehnen, arXiv:1604.05493 [Phys. Rev. D (to be published)].

[20] J. Oñorbe, M. Boylan-Kolchin, J. S. Bullock, P. F. Hopkins, D. Kereš, Claude-André Faucher-Giguère, E. Quataert, and N. Murray, Mon. Not. R. Astron. Soc. 454, 2092 (2015).

[21] J. I. Read, O. Agertz, and M. L. M. Collins, arXiv: 1508.04143

[22] J. F. Navarro, C. S. Frenk, and S. D. M. White, Astrophys. J. 490, 493 (1997).

[23] J. Peñarrubia, A. Pontzen, M. G. Walker, and S. E. Koposov, Astrophys. J. Lett. 759, L42 (2012).

[24] J. Peñarrubia, A. J. Benson, M. G. Walker, G. F. Gilmore, A. W. McConnachie, and L. Mayer, Mon. Not. R. Astron. Soc. 406, 1290 (2010).

[25] A. Agnello and N. W. Evans, Astrophys. J. Lett. 754, L39 (2012).

[26] M. G. Walker, M. Mateo, E. W. Olszewski, J. Peñarrubia, N. W. Evans, and G. Gilmore, Astrophys. J. 704, 1274 (2009).

[27] J. Wolf, G. D. Martinez, J. S. Bullock, M. Kaplinghat, M. Geha, R. R. Munoz, J. Simon, and F. Avedo, Mon. Not. R. Astron. Soc. 406, 1220 (2010).

[28] M. G. Walker and J. Peñarrubia, Astrophys. J. 742, 20 (2011).

[29] A. Boyarsky, O. Ruchayskiy, D. Iakubovskyi, M. G. Walker, S. Riemer-Sørensen, and S. H. Hansen, Mon. Not. R. Astron. Soc. 407, 1188 (2010).

[30] L. Hernquist, Astrophys. J. 356, 359 (1990).

[31] N. W. Evans and A. A. Williams, Mon. Not. R. Astron. Soc. 443, 791 (2014).
[32] A. W. McConnachie, Astron. J. 144, 4 (2012).

[33] E. N. Kirby, M. Boylan-Kolchin, J. G. Cohen, M. Geha, J. S. Bullock, and M. Kaplinghat, Astrophys. J. 770, 16 (2013).

[34] N. F. Martin, D. L. Nidever, G. Besla et al., Astrophys. J. Lett. 804, L5 (2015).

[35] V. Belokurov, M. G. Walker, N. W. Evans et al., Astrophys. J. Lett. 712, L103 (2010).

[36] E. N. Kirby, J. D. Simon, and J. G. Cohen, Astrophys. J. 810, 56 (2015).

[37] M. G. Walker, M. Mateo, E. W. Olszewski et al., arXiv:1511.06296.

[38] N. C. Amorisco and N. W. Evans, Mon. Not. R. Astron. Soc. 411, 2118 (2011).

[39] V. Bonnivard, C. Combet, M. Daniel et al., Mon. Not. R. Astron. Soc. 453, 849 (2015).

[40] M. Ackermann et al., Phys. Rev. D 89, 042001 (2014).

[41] V. Bonnivard, D. Maurin, and M. G. Walker, arXiv: 1506.08209 .

[42] S. E. Koposov, G. Gilmore, M. G. Walker et al., Astrophys. J. 736, 146 (2011).

[43] M. Niederste-Ostholt, V. Belokurov, N. W. Evans, G. Gilmore, R. F. G. Wyse, and J. E. Norris, Mon. Not. R. Astron. Soc. 398, 1771 (2009).

[44] B. Willman, M. Geha, J. Strader, L. E. Strigari, J. D. Simon, E. Kirby, N. Ho, and A. Warres, Astron. J. 142, 128 (2011).

[45] K. Bechtol, A. Drlica-Wagner, E. Balbinot et al., Astrophys. J. 807, 50 (2015).

[46] S. E. Koposov, A. R. Casey, V. Belokurov et al., Astrophys. J. 811, 62 (2015).

[47] J. T. Kleyna, M. I. Wilkinson, G. Gilmore, and N. W. Evans, Astrophys. J. Lett. 588, L21 (2003).

[48] D. R. Cole, W. Dehnen, J. I. Read, and M. I. Wilkinson, Mon. Not. R. Astron. Soc. 426, 601 (2012).

[49] N. C. Amorisco and N. W. Evans, Mon. Not. R. Astron. Soc. 419, 184 (2012).

[50] N. C. Amorisco, A. Agnello, and N. W. Evans, Mon. Not. R. Astron. Soc. 429, L89 (2013).

[51] H. Zhao, Mon. Not. R. Astron. Soc. 278, 488 (1996).

[52] J. Han, W. Wang, S. Cole, and C. S. Frenk, Mon. Not. R. Astron. Soc. 456, 1003 (2016). 